\title{
Translation of English and Chinese Addressing Terms from the Cultural Aspect
}

\author{
Chunli Yang \\ Foreign Languages School, Zhengzhou University, Zhengzhou, China \\ Email: Sunday1979@126.com
}

\begin{abstract}
Terms of address are frequently used in social communication to show the identity, rank and relationship between the speaker and the hearer. It is important to have a profound understanding of addressing terms, especially in cross-cultural communication. However, due to the difference in history, religion and culture, terms of address vary in different countries and regions. In this paper, the author discusses the translation of English and Chinese addressing terms from the aspect of cultural background. First, the author defines terms of address and gives the reason why people use terms of address in social communication. Then, the differences between English and Chinese terms of address are analyzed from the point of kinship terms and social terms of address. Last, four translation methods are given according to different situations, namely, literal translation, translating flexibly, specification or generalization and domestication and foreignization.
\end{abstract}

Index Terms - terms of address, kinship terms, social terms of address, translation methods

\section{INTRODUCTION}

With the development of globalization and the expansion of the intercultural communication, the contact between people from different countries, cultures and races are becoming more and more frequent. In the communication, it is inevitable to use terms of address which differ because of the diversity of culture. Moreover, terms of address are culture dependent and change in the course of time as old criteria become obsolete and come to be replaced by new criteria, and other criteria may be added to create a new system (Taavitsainen and Jucker, 2003). During thousands of years' development, China has formed a very sophisticated address system, while the addressing terms in western countries are relatively simple. There are seldom absolute address equivalent address terms in Chinese and English. Therefore, in order to make the translation more precisely and specify the exact relation between people, translators should have a profound understanding of various terms of address. In this paper the reason for differences existed between Chinese and English terms will be discussed and corresponding translation methods will be given.

\section{TERMS OF ADDRESS}

Terms of address are words and phrases used for addressing. They refer to the collocutor and thus contain a strong element of deixis (Braun, 1988). They are words or linguistic expressions that speakers use to appeal directly to their addressees (Taavitsainen and Jucker, 2003). In Modern Chinese Dictionary, address terms are defined as terms people get because of kinship or other relationship as well as their status, occupation, etc, such as 'father', 'master', and 'boss'.

To be brief, terms of address are words or expressions used to indicate certain relations between people, or to show the difference in identity, position and social status. They are reflections of national cultures. It plays a very important role in the face-to-face communication since it is the first information transferred to others.

It is true that people use terms of address to address each other in almost all occasions. However, it is also true that sometimes it is not necessary to use them when people involved in the face-to-face communication know each other well. Yet, people still use them even in those situations. Why? First, they are used to attract people's attention, to remind the hearer one's professional status or the relationship between the speaker and the hearer. Second, they are used to show politeness and the difference in social class and the degree of respect in certain occasions. Third, they are used to reflect social information about identity, gender, age, status and the complex social relationships of interlocutors in a speech community.

As there are so many terms of address, they can be classified into different categories according to different criteria. In A Dictionary of Epithets and Terms of Address, Dunkling divides it into two types, nominal vocatives and verbal vocatives (Dunkling, 1990). While Braun classifies it into pronouns of address, verb forms of address, nouns of address and bound forms of address and free forms of address (Braun 1988).

Different from those, in this paper the author classifies it into two categories: kinship terms and social terms of address. Kinship terms are terms for blood relations and for affine (Braun, 1988), indicating the relationship between one person and his relatives. This kind of terms is mainly used between family members. Social terms of address refer to all addressing terms expect kinship terms, used in social communications reflecting the social relations, status and level of respect between the speaker and the addressee in the communication. In A Preliminary Study of the Translation 
of Chinese Appellations into English, social terms of address are divided into six categories: person terms of address, title terms of address, general terms of address, and profession terms of address, kinship terms referring to non-relatives and context terms of address.

\section{DIFFERENCE BETWEEN ENGLISH AND CHINESE TERMS OF ADDRESS}

Terms of address may differ according to the formality of the situation, the social relationship between the speaker and the addressee, the politeness or deference that the speaker wants to extend to the addressee (Taavitsainen and Jucker, 2003). It may differ because of the difference in history, social structure and culture. In this part, the reasons why Chinese and English kinship terms and social terms of address differ will be analyzed respectively.

\section{A. Difference in Kinship Terms}

During the development of human history, differences in political system and ideology have caused the distinct in culture and traditional ethics. As a result, the kinship terms in Chinese and English differ mainly due to the distinct of social ethics, blood relationship and traditional customs.

Social ethics have a significant impact on the formation of kinship terms. As we all know that China has several thousand years of feudal history, the whole nation was supported by hundreds of thousands of families. The 'family' in China has traditionally been the centre of all activity and is the focus of Chinese life (Methven, 2006). Generation and age are regarded as important elements when addressing others in the same family.

On the one hand, generation has long taken root in Chinese's traditional ideas. There has been nine generations in a family since ancient China, namely, gao zu, zeng $z u$, zu, fu, ben ren, zi, sun, zeng sun, xuan sun. However, in western countries, the nuclear family is the core of the whole society with at most three generations living in a family. Therefore, kinship terms in English are much simpler since generation is not taken seriously. There are only three expressions in terms of generations, that is, grandfather, father, son and grandson. If one wants to express gaozu, zengzu, zengsun, xuansun, "great" or "great great" should be added to the terms, such as great great grandfather, great grandfather, great grandson and great great grandson.

On the other hand, age is considered as another significant factor in determining the choice of address terms in Chinese. "Zhang you you xu" is highly valued in Chinese culture, so the senior has priority over the junior. Whereas, westerners believe that everyone is born equal, so they pay little attention to the difference in age. Numbers are commonly seen in Chinese address terms to show the order. For example, 'da ge', 'er di', san yi', 'si gu' are used to show the difference in generation and age (Bao Huinan, 2001). However, there is no such kind of usage in English. "Brother" can refer to both one's younger and older brother, while "sister" can refer to one's younger and older sister. Westerners pay little attention to age; they usually address each other by the name. It is the same even between the younger generation and the elder generation.

Blood relationship also affects the shaping of kinship terms which makes the kinship terms in Chinese rather complex than those in English. Influenced by the feudal ethics, Chinese established a strict clan system based on blood relationship. In one clan, there may be tens of, hundreds of or even thousands of members living together with quite complex relations. In Chinese, there are two kinds of relatives. One is blood relatives which indicate relatives with blood relations. The other is adfinis which refers to relatives formed by marriage (Bao Huinan, 2001). Therefore, terms addressing relatives on the father's side are different from those used to address relatives on the mother's side. For instance, "shu shu"and "gu gu" are paternal relatives while "jiu jiu "and"yi" are maternal relatives. However, in western countries, individualism is paid much emphasis on. Only children under age live with their parents in a nuclear family, while the grown-up children will leave home and rely on their own efforts. This makes the family members in a small number. Therefore, family address terms in English are very simple, based around the nuclear family where there is less need to distinguish between distant relations (Methvon, 2006). For example, "Uncle" is used to refer to both the younger or older brother of the paternal side and the younger or older brother of the maternal side.

The distinct in traditional customs is another factor that impact kinship terms. Males and females have been seen to be different since ancient China, namely, "nan nv you bie", which means that address terms referring to males and females are absolutely different. The typical instance is the meaning of "cousin" which can express eight address terms in Chinese, namely, "biao ge, biao di, biao jie, biao mei ,tang ge, tang di, tang jie, tang mei". In English, children of the parents' brothers and sisters can be addressed by "cousin", no matter which gender they belong to and no matter how old they are. While in Chinese "tang ge, tang di, biao ge, biao di" can only refer to males, while "tang jie, tang mei, biao jie, biao me" only refer to females.

\section{B. Difference in Social Terms of Address}

In social communication, it is inevitable to use social terms of address to show politeness or respect. Therefore, it is very important to understand different meanings that social terms of address have, especially in inter-cultural communication. Social terms of address are greatly affected by social class and traditional ethical concept which are essential parts in deciding which term to use.

Chinese people appear to have a very strong sense of position in the hierarchical social structure and try to keep interpersonal harmony by obeying the prescribed order in their everyday social interaction (Xianghong, 2006). 
Therefore, people used to employ honorific expressions to refer to those who have a higher status and modest expressions to themselves. For instance, "dian xia, fu ren, da ren" etc are used to address those having a high rank, while "xia guan, xiao ren, nu bi" etc are terms for those with low ranks.

In ancient Chinese culture, politeness was considered to be an inner quality that was expressed outwardly through the use of honorific language, whereby the speaker would use self-deprecating terms to refer to him/herself and related people (or place), and use respectful terms to refer to others (Yanping 2004 qtd Methven, 2006).

For example, "ling", "xian" and "lao" are often added to addresses to show respect, such as "lao tai tai", "ling lang”, "xian di", etc. Meanwhile, "bi ren”, "wan sheng”, "xue sheng” are used to refer to oneself. In western countries, people always emphasize on democracy and equality, so names are often used directly to address each other, even though they are not in the same generation.

There is another situation unique to China that kinship terms are used to refer to those who are not their relatives. China is a country that regards courtesy as the foundation stone of its traditional ethics, and in the Chinese mentality, society as a whole is seen as an extension of the family, and there is no clear distinct between family and country (Methvon, 2006). People usually use "da ye, da niang", "shu shu", "a yi" to address strangers just out of courtesy and shorten the distance between them while communicating. Under the influence of individualism, westerners think highly of personal ability and achievement more than relationship. They seldom use kinship terms to refer to non-family members because it may be considered quite rude. For example, addressing an older women "grandma" might cause offense, since in western cultures, "old" means aged and useless. On the contrary, they usually use general social terms of address to refer to those non-family members.

\section{TRANSLATION MethodS}

As one can see from the above, due to the difference between Chinese and English cultures, terms of address in Chinese and English are quite different. The address system in Chinese is sophisticated with various terms while the address system in English is comparatively simple. This difference inevitably brings difficulty to translation. In order to respect traditional expressions in one culture and make readers clearly understand the difference between Chinese and English addressing terms, translators should master certain translation skills.

\section{A. Literal Translation}

When doing literal translation, translators do their best to remain as many as possible the ideas, sentences structures and writing style of the source language. It is the simplest way in translation, but only a few terms of address can be translated by this method.

In English kinship terms, father, mother, son and daughter are the only terms that can be translated literally into " $f u$ qin, mu qin, er zi, nv er", and vice versa.

Due to the fact that Chinese social terms of address far outnumber those in English, there are a few expressions of general terms and certain terms indicating one's profession and title that can be translated literally. The equivalence of "xian sheng, fu ren, xiao jie" which are frequently used in Chinese can be found in English as "Sir.", "Mrs." and "Miss".

There are only several terms that can indicate one's profession in English, such as "President, judge, doctor, officer", etc. Terms reflecting one's title are Dr, Prof, President, General, Captain, Pop, etc. For example,

Doctor White Professor Smith

President Clinton Captain Jack

General John Pop William

\section{B. $\quad$ Translating Flexibly}

Terms of address in Chinese are complex and numerous in numbers, while address terms in English are comparatively simple and in a small number. One term indicates a certain people in Chinese while one word may refer to several people in English. This makes it easy to translate Chinese address terms into English. Also, due to this, context should be considered while translating English address terms into Chinese. That is, one must, according to the context, convert the terms of address of the source language to the corresponding or similar terms of address of the target language in order to adjust to the traditional expression of the target language. For example:

'Not aunt,' cried Oliver, throwing his arms about her neck; 'I'll never call her aunt - sister, my own dear sister, that something taught my heart to love so dearly from the first! Rose, dear, darling Rose!'

" nib u shi xiao yi", Olive shuang bi lou zhu le ta de bo zi jiao dao, "wo zen me ye bu jiao ni yi--ni shi wo jie jie, wo zui qin ai de qin jie jie,wo cong yi kai shi jiu na me de ai ni, na me de xi huan ni! Rose, qin ai de, qin ai de Rose!"

When translating English kinship terms, one should consider the context. In the above example, one has to think of the whole relation since aunt has more than one meaning, such as " $g u$ gu yi ma, jiu mu, bo mu" etc. Conversely, it is much easier to translate Chinese kinship terms into English since all sisters and spouses of one's parents' brothers are called aunt.

Since kinship terms can be used to refer to those who are not one's relatives in Chinese, to address people, one can use kinship terms directly, such as: ye ye, shu shu, a yi, etc. or use xiang/da/lao+kinship terms, such as zhang shu, da 
niang, lao bo, etc. However, in western countries, there is no such kind of usage, so it must be highly noted that this kind of addresses should be appropriately converted to traditional expressions in English.

nv tong dao: " ye ye de bing qi, bu zai zhe li." Niu wang dao: " na ni nai nai de bing qi lai ba!"

"Your Majesty's weapons aren't here," the serving girls replied. "Then bring your mistress' weapons," the Bull Demon King replied. (Journey to the West P1996)

The translation of honorific terms is also a difficulty. There are several ways to show the respect to the other person involved in the communication. First, using lao/xiao/xing to show intimacy and shorten the distance, which are mainly used by the senior to refer to the junior or among people in the same generation such as lao wang, xiao li. It is generally translated into Old/Little +Family name, such as Old Wang, Little Li. Then, using xing +lao to show respect, such as: guo lao, xue lao, etc. Last, using special terms, such as :lao shi, fu ren, xian sheng, etc. For instance:

"xian sheng he bi guo qian. Jiu qing xian sheng jin qu kan kan er fu, yang zhang gao ming, yi shi xia wai."

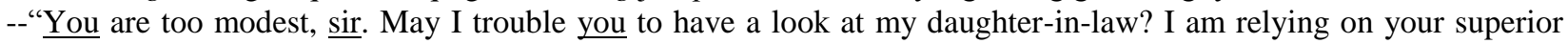
knowledge to relieve our anxiety." (Yang Xianyi \& Gladys Yang, 2003)

\section{Specification or Generalization}

Chinese often use vague address terms to refer to certain people. For example, women use "wo men jia na wei"," $n i$ ge si gui",etc to address their husbands. In that case, translators should point out the specific person it refers to. Moreover, the original work sometimes uses too many addresses indicating many different persons who are not closely related to the development of the main plot. Under these circumstances, there is no need to translate these addresses one by one. They can be generalized in order to make the translated version more concise and easily understood. For example,

"zhen ge shao you, bie ren bu guo shi li shang mian zi qing, shi zai ta shi zhen teng xiao shu zi, xiao gu zi. Jiu shi zai lao tai tai gen qian, ye zhen shi xiao shun."

-- "Other young married women do more than politeness requires, whereas she has genuine feeling for her husband's younger relatives and is truly dutiful to you as well, madam." (Yang Xianyi \& Gladys Yang, 2003)

Here, "xiao shu zi", "xiao gu zi" refer to Jia Lian's sisters and brothers on the father's side. Take consideration of the context, these two terms of address are used to compliment Xifeng on her kindness. Therefore, generalization should be used to make the plot much simpler.

\section{Domestication and Alienation}

Domestication and foreignization are two important strategies in the translation of Chinese terms of address into English. Domestication means an ethnocentric reduction of the foreign text to target-language cultural values (Venuti, 1995 qtd Munday, 2006). This entails translating in a transparent, fluent, 'invisible' style in order to minimize the foreignness of the TT. Foreignization refers to an ethnodeviant pressure on 'target-language cultural'values to register the linguistic and cultural difference of the foreign text, sending the reader abroad.

In other words, domestication is, in the process of translation, using as much as possible local ways to express the ideas of the foreign work. Foreignization, on the other hand, means remaining as much as possible the language and culture in foreign works. In short, domestication is reader-oriented while foreignization is author-oriented.

Terms of address indicating age differences are constantly used in Chinese, while address terms referring to one's name are frequently employed. Therefore, in translating Chinese address terms, one can use the method of forengnization to translate them into English name terms. In addition, foreignization can also be used to explicitly indicate certain person when the relations between people in Chinese are quite complicated.

Zhou rui jia de da ying le, yin shuo, "si gu niang bu zai fang li, zhi pa zai lao tai tai na bian ne."

-- As Mrs. Zhou handed over the flower she remarked, "The fourth young lady isn't here. Is she with the old lady, I wonder?" ( Yang Xianyi \& Gladys Yang, 2003)

In this sentence, "Zhou rui jia"refers to the wife of Zhourui, so specification is used to mean Mrs. Zhou. Meanwhile, there is no such expression as "si gu niang"in English. In order to make clear who it really indicates, it has to be translated in a more Chinese way. That is why foreignization is used to translate it into "the fourth young lady".

Furthermore, honorific terms of address and modest address terms are frequently used to show respect and modest during the communication. There are many modest address terms in Chinese which can inflect the difference in rank, identity, profession, gender, etc. Bao Huinan divided them into the following categories (Bao Huinan, 2001):

1) Modest terms indicating ranks: "zhen, gu, gua"are for monarch; "wei chen, xiao li, xia guan"are for officials; "xiao min, cao min, min fu"are for the common people; etc.

2) Modest terms indicating identity: "nu cai, xia ren, xiao de" are for servants; "wan sheng, xiao di, di zi, yu sheng"are for students, etc.

3) Modest terms indicating gender: "qie, jian qie, nu jia, jian qi, jian nei"are for women, etc.

4) Modest terms indicating profession: "pin dao, xiao dao, pin seng"are for monks, etc.

Except these, modest address terms are also used to address one's family members and relatives. For example, "jia fu, jia xiong, nei ren, quan zi", etc. refer to respectively one's father, elder brother, wife and son.

In English, "I" and "we" are used to refer to oneself. Only in very rare occasions are the terms "one" or "yours truly" employed. Hence, domestication should be used while doing Chinese-English translation. Yet, the time of the work, the 
identity of the character and the feature of the literary form should be taken into consideration when translating English modest address terms into Chinese. For example:

Xiang ba, you dao, " xian shi ji bu ken shuo po qian yin, di zi yu xin he ren?"

--“How can I bear it, saintly teacher," he said, "if you draw a veil over your past?" (Yang Xianyi \& Gladys Yang, 2003, P3103)

Using honorific terms of address is a tradition in China during contact. As for the elders who have official titles, "lao, shi"should be added to the address term, such as "lao xian sheng"、 "lao tai tai" " "shi weng", etc. As for one's teacher and his wife, "xian shi, en shi, shi mu, shi niang", etc. are used. As for other's children, "ling" has to be employed, such as "ling yuan, ling lang, ling qian jin", etc. "shi xiong, shi xiong, xian di, xian mei" are also commonly used to refer to non-relatives.

Only "you, he, she and they" are used to address others in English address terms. For the royalty, the nobility and officials with higher ranks, "Your/ His/ Her Highness/ Honor/ Lordship/ Ladyship/ Majesty" or "My Lord" is the common terms of address. In most occasions, when translating honorific terms of address, it is unlikely the translator will find a semantic equivalent where the cultural content of a term can be entirely rendered into the target text (Methvon, 2006). Therefore, the best way is using domestication to make the address acceptable in the target language. For instance:

- Do not, my lord.

-Why, what should be the fear? I do not set my life at a pin's fee. (Shakespear, 1991 )

- Bu yao qu, dian xia.

-Hai, pa shen me ne? wo ba wo de sheng ming kan de yi qian bu zhi

\section{CONCLUSION}

This paper mainly shows the difference between Chinese and English terms of address and the reasons why these differences exist. Due to the difference between Chinese and western cultures, people have different traditional ethics and customs. Under the influence of thousands of years' feudal system and traditional ethics, China has become a nation based on large clans which are established on the basis of blood relationship. In the complicated clan system, generation, age and gender are regarded essential factor determining the choice of address terms. However, western countries have a relatively short history during which democratic countries were founded advocating democracy, equality and individualism. People lay less on generation, age and blood relationship which contribute to the simplicity of English kinship terms.

In the society, people use different social terms to address others. The formation of these address terms are influenced by social class, traditional ethics and politeness. Due to this, Chinese social terms of address far outnumber and are much more complex than those in English.

Then, in order to make the translated version more precise and make readers understand easily, four translation methods are given according to different situations, namely, literal translation, translating flexibly, specification or generalization and domestication and foreignization.

\section{REFERENCES}

[1] Braun, Friederike. (1988).Terms of Address: Problems of Patterns and Usage in Various Language and Cultures. Berlin: Mouton de Gruyter .

[2] Chunjie, Qiao. (2009). On the Translation of Address Terms in Hongloumeng from the Perspective of Skopostheorie. Zhengzhou: Zhengzhou University Press.

[3] Dunkling, L.A. (1990). Dictionary of Epithets and Terms of Address. USA and Canada: Routledge.

[4] Methven, Andrew. (2006). Discussion of the Difficulties in Translating Terms of Address in Chinese and English. MA Chinese Translation Practice and Theory.

[5] Munday, Jeremy. (2001).Introducing Translation Studies: Theories and Applications. Oxon: Routledge.

[6] Ruirong, Wang. (2003). A Preliminary Study of the Translation of Chinese Appellations into English. Journal of Southwest Agricultural University (Social Science Edition), Vol. 1, No. 4.

[7] Taavitsainen, Irma and Jucker, H. Andreas. (2003). Diachronic Perspective on Address Term Systems. Philadelphia: John Benjamins North America.

[8] Xianghong, Cao. (2006). A Pragmatic Analysis of Address Forms in Chinese Family and Non-family Letters. China Media Research, 2 (1) : 60.

[9] Yang Xianyi \& Gladys Yang trans. (2003). A Dream of Red Mansions. Beijing: Foreign Languages Press.

[10] Shakespeare, (1991). Hamlet. Beijing: Hua Wen Press.

[11] Bao Huinan. (2001).Cultural Context and Language Translation. Beijing: China Translation\& Publishing Corporation.

Chunli Yang was born in Henan, China in 1974. She received her M.A. degree in linguistics from Peking University, China in 2004.

She is currently a lecturer in the School of Foreign Languages, Zhengzhou University, Zhengzhou, China. Her research interests include translation studies and linguistics. She is a member of Henan Translation Association. 